\title{
Atomic noise spectra in nonlinear magneto-optical rotation in a rubidium vapor
}

\author{
Hebin Li, ${ }^{1}$ Vladimir A. Sautenkov, ${ }^{1,2}$ Tigran S. Varzhapetyan, ${ }^{1,3}$ Yuri V. Rostovtsev, ${ }^{1}$ and Marlan O. Scully ${ }^{1,4}$ \\ ${ }^{1}$ Institute for Quantum Studies and Department of Physics, \\ Texas AEM University, College Station, Texas 77843, USA \\ ${ }^{2}$ Lebedev Institute of Physics, Moscow 119991, Russia \\ ${ }^{3}$ Institute for Physical Research of NAS of Armenia, Ashtarak-2 378410, Armenia and \\ ${ }^{4}$ Princeton Institute for the Science and Technology of Materials and Department of Mechanical E Aerospace Engineering, \\ Princeton University, Princeton, New Jersey 08544, USA
}

\begin{abstract}
We have studied the noise spectra in a nonlinear magneto-optical rotation experiment in a rubidium vapor. We observed the reduction of noise in the intensity difference of two orthogonally polarized components of the laser beam. The dependence of the noise level on both the frequency and the longitudinal magnetic field has been studied. We found that the optimal condition for the noise reduction is to work around zero longitudinal magnetic field, where the intensity correlation between the two orthogonally polarized components is maximum. Our results can be used to reduce or eliminate the atomic excess noise, therefore improving the sensitivity of nonlinear magneto-optical rotation magnetometers and other atom-optical based applications.
\end{abstract}

\section{INTRODUCTION}

Improving the sensitivity of magnetometers is important both for practical applications and for fundamental research. Magnetometers based on atom-optical techniques, such as the optical pumping magnetometers [1] and the nonlinear magneto-optical rotation (NMOR) magnetometers [2, 3, 4], have achieved sensitivities of the order of $10^{-15} \mathrm{~T} \mathrm{~Hz}^{-1 / 2}$. Quantum noise starts to play a crucial role in obtaining higher sensitivity, which approaches the atom shot-noise-limited sensitivity [5]. One of the contributions to the quantum noise is the increase of laser beam intensity fluctuation due to the laser interacting with an atomic vapor (atomic excess noise). Possible processes that are responsible for the generation of the atomic excess noise include the conversion of laser phase noise to intensity noise [6, 7, 8, 9] and the four-wave mixing process 10]. Although the atomic excess noise could be a useful spectroscopic tool [6, 11], it is usually not desirable in atom-optical based applications, such as atomoptical magnetometers, atomic frequency references 12], and the generation of squeezed light [13, 14, 15, 16, 17].

To reduce or eliminate the influence of atomic excess noise, one can take advantage of the intensity correlation properties of the optical fields passing through an atomic vapor. As is shown in [18], an electromagnetically induced transparency (EIT) experiment was performed by coupling two beams from one laser with an excited state and Zeeman sublevels of the ground state in a rubidium vapor. The authors observed the intensity correlation and anticorrelation between two circularly polarized laser beams. More generally, a similar effect was also observed with two beams from two independent lasers [19]. In the case of correlation or anticorrelation, the intensity noise in each of two laser fields is fluctuating with a phase difference of 0 or $180^{\circ}$. A simple summation or subtraction of these two signals can suppress the noise. Experiments have shown the ability of reducing the noise to the shotnoise level by taking the difference of two laser beams in an EIT configuration [20]. In NMOR experiments, the recent observation of the intensity correlation 21], along with the power spectra study of the noise at $2.5 \mathrm{MHz}[22]$, indicate the possibility of using the intensity correlation to reduce or eliminate the atomic excess noise.

In this paper, we report the experimental study of the noise spectra in a nonlinear magneto-optical rotation experiment in a rubidium vapor. We show that the atomic excess noise in NMOR can be essentially reduced to the shot-noise level because of the intensity correlation of two orthogonally polarized components. The dependence of the noise in the difference signal of these two components on both frequency and magnetic field, has been studied. Our results show that the optimal working condition for reducing the atomic excess noise is to work near zero longitudinal magnetic field.

\section{EXPERIMENTAL SETUP}

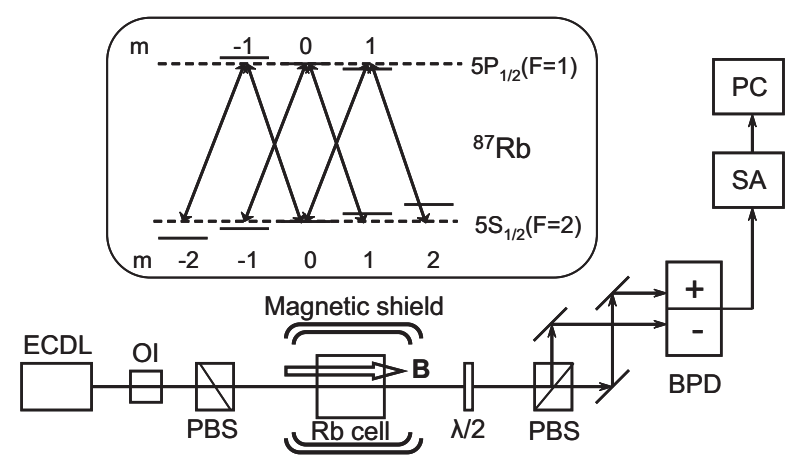

FIG. 1: The experimental schematic and the energy diagram (the inset). ECDL: external cavity diode laser; OI: optical isolator; PBS: polarizing beam splitter; $\lambda / 2$ : half-wave plate; BPD: balanced photo diode; SA: spectrum analyzer; PC: computer.

The experimental schematic is illustrated in Fig. 1. The laser source is an external cavity diode laser (ECDL) 
described in [23]. The laser is tuned to the rubidium $\mathrm{D}_{1}$ line $(795 \mathrm{~nm})$, specifically at the transition $5 \mathrm{~S}_{1 / 2}(\mathrm{~F}=2)$ $\leftrightarrow 5 \mathrm{P}_{1 / 2}(\mathrm{~F}=1)$ of ${ }^{87} \mathrm{Rb}$, referenced to the Doppler-free saturation resonance in a rubidium cell at room temperature. The frequency drift is less than $30 \mathrm{MHz}$ per hour after a sufficient warm-up time. The linewidth of the laser emission is less than $1 \mathrm{MHz}$. The laser beam has a diameter of $1 \mathrm{~mm}$, and it is linearly polarized. After passing through an optical isolator, the beam proceeds through a polarizing beam splitter (PBS) and possesses a polarization parallel to the optical table.

The beam goes into a glass cell filled with a rubidium vapor that contains the natural isotope abundance of rubidium atoms. The cell has the length of $7.5 \mathrm{~cm}$, and it is heated to reach an atomic density of $10^{12} \mathrm{~cm}^{-3}$. A two-layer magnetic shield isolates the cell from environmental magnetic fields in the lab, while a solenoid inside the magnetic shield provides an adjustable longitudinal magnetic field. The linearly polarized beam is a combination of the left- and right-circularly polarized components. The two circular components are coupled to the energy levels of ${ }^{87} \mathrm{Rb}$ as shown in the energy diagram in Fig. 1.

The output beam from the rubidium cell is analyzed by a half-wave plate $(\lambda / 2)$ and a PBS. The half-wave plate is set to rotate the polarization by $45^{\circ}$, such that without the rubidium cell, the PBS equally splits the intensity of the beam. If the rubidium cell is placed in the system, a rotation angle of the beam polarization will be introduced that depends on the magnitude of the longitudinal magnetic field [5]. With a nonzero magnetic field $(B \neq 0)$, the two beams coming out from the PBS do not have equal intensities. Recording the intensities of two beams as $I_{1}$ and $I_{2}$, the polarization rotation due to rubidium atoms can be calculated using the following equation

$$
\phi=\arcsin \left(\frac{I_{1}-I_{2}}{I_{1}+I_{2}}\right) .
$$

To study the power spectra of the atomic excess noise, a balanced photo detector (BPD) with a sensitivity of $2 \times 10^{4} \mathrm{~V} / \mathrm{W}$ and a bandwidth from DC to $100 \mathrm{MHz}$ is used to register the intensities of two laser beams. The optical path lengths of the beams going into two channels of the BPD are chosen to be the same so that no additional time delay between the two channels is introduced. The signal is analyzed by an RF spectrum analyzer. In the case of a zero magnetic field $(\mathrm{B}=0)$, for example, each channel of the $\mathrm{BPD}$ records an intensity

$$
I_{i}=I_{0}+I(t)+\delta I_{i}(t),(i=1,2),
$$

where $I_{0}$ is the average intensity, $I(t)$ is the low frequency intensity fluctuations, and $\delta I_{i}(t)$ is the atomic excess noise. Then, the difference signal $\Delta I$ from the BPD is given by $\Delta I(t)=\delta I_{1}(t)-\delta I_{2}(t)$. The spectrum analyzer gives the Fourier transform of the time dependence of the signal.

\section{EXPERIMENTAL RESULTS AND DISCUSSION}
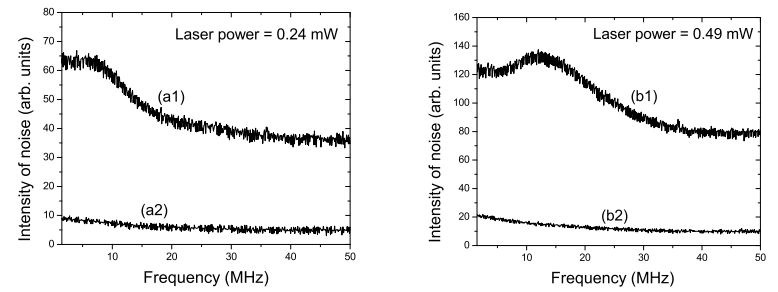

FIG. 2: The power spectra of the noise from laser beams in an NMOR experiment with the magnetic field $B=0$. The left and right figures show the spectra with input laser powers of $0.24 \mathrm{~mW}$ and $0.49 \mathrm{~mW}$, respectively. Traces (a1) and (b1) are the noise spectra of one laser beam. Traces (a2) and (b2) are the noise spectra of the balanced signal (with both beams). The spectrum analyzer was setup with a resolution of $300 \mathrm{kHz}$ and a video bandwidth of $100 \mathrm{~Hz}$.

We begin the presentation of the results by showing the power spectra of noise with no magnetic field $(\mathrm{B}=0)$. Figure 2 shows the noise spectra for different input laser intensities. The left and right figures display the spectra for input laser powers of $0.24 \mathrm{~mW}$ and $0.49 \mathrm{~mW}$, respectively. Traces (a2) and (b2) are recorded with two laser beams sent to the BPD, and they show the noise spectra of the difference signal. The noise is larger in the low frequency region. The noise level approaches the shot-noise level [20] at higher frequencies. For comparison, traces (a1) and (b1) are recorded with only one laser beam sent to the BPD. They represent the noise spectra of the laser beam passing through the rubidium vapor. Before entering the cell, the laser beam has small intensity noise but large phase noise. The phase noise is converted into the intensity noise due to the laser interacting with the atoms. This process causes a substantial increase of intensity fluctuations in the laser beam coming out of the cell [6]. Our results show that these intensity fluctuations can be suppressed by subtracting the intensity of one laser beam from the other. Comparing the noise spectra of one laser beam and of the difference signal, (a1) and (a2) for instance, the noise level of the difference signal is dramatically reduced. When the input laser power is doubled $(0.49 \mathrm{~mW})$, the corresponding spectra presented as (b1) and (b2) show the same behavior, although the shot-noise level increases approximately two times because of the higher laser power. These results can be understood as a consequence of the intensity correlation between the two output laser beams from the PBS. As is shown in [21], the intensities of two beams in an NMOR experiment are highly correlated (correlation function $\left.G^{(2)}(0) \approx 0.9\right)$ at zero magnetic field $(\mathrm{B}=0)$. The fluctuations $\delta I_{1}(t)$ and $\delta I_{2}(t)$ are varying simultaneously, and thus $\Delta I(t)$ will be small.

The intensity correlation and the substantial reduction 


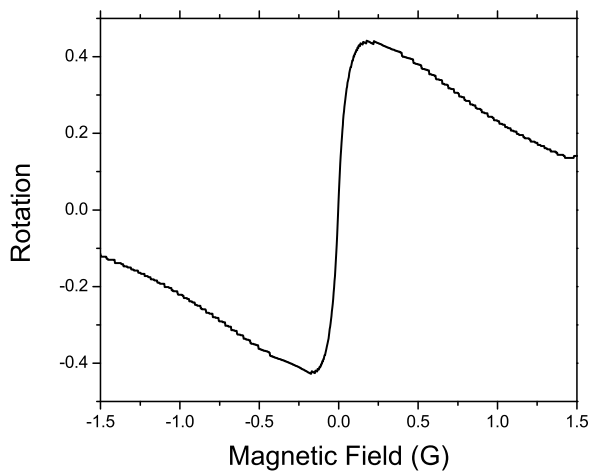

FIG. 3: The polarization rotation is plotted as a function of the longitudinal magnetic field B.

of noise in NMOR experiments is not trivial in terms of various behaviors at different magnitudes of the magnetic field $\mathrm{B}$. To show this, besides the preceding results with a zero magnetic field, we have also studied the noise spectra in NMOR at magnetic fields of various magnitudes. Prior to showing these results, a typical measurement of the polarization rotation in our experiment (laser power $\mathrm{P}=0.24 \mathrm{~mW}$ ) is presented in Fig. 3, to remind us of the rotation dependence on the magnetic field.
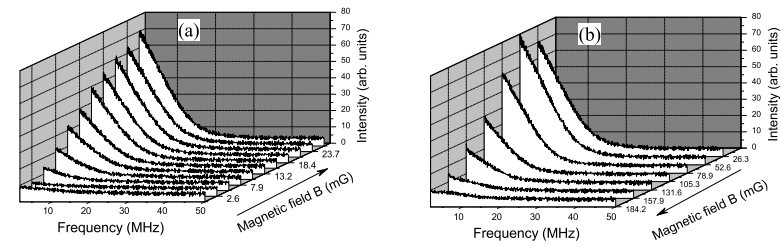

FIG. 4: The noise spectra dependence on the longitudinal magnetic field. The noise level is plotted as a function of both the frequency and the magnetic field. (a) The spectra corresponding to magnetic fields ranging from 0 to $26.3 \mathrm{mG}$; (b) The the spectra corresponding to magnetic fields ranging from $26.3 \mathrm{mG}$ to $184 \mathrm{mG}$. The arrows denote the ascending direction of the magnitude of the magnetic field.

We record the noise spectra of the difference signal with two output beams sent to the BPD for several magnetic fields. The results are presented as three dimensional plots in Fig. 4 (laser power $\mathrm{P}=0.24 \mathrm{~mW}$ ). The magnetic field varies from 0 to $184 \mathrm{mG}$. The spectra corresponding to magnetic fields ranging from 0 to $26.3 \mathrm{mG}$ are shown in plot (a), and the ones corresponding to magnetic field ranging from $26.3 \mathrm{mG}$ to $184 \mathrm{mG}$ are shown in plot (b). The spectra are sorted by the magnetic field ascending along the arrows shown in the figure. The magnetic field in plot (a) steps by about $2.6 \mathrm{mG}$, while it steps by about $26 \mathrm{mG}$ in plot (b). Note that the noise spectra corresponding to negative magnetic fields, which are not plotted here, have the symmetric behaviors. From these results, we see that the reduction of high frequency noise is nearly the same for different magnetic fields, but the low frequency noise is not appreciably reduced. This shows that the low frequency noise is not correlated at all magnitudes of the magnetic field, but the high frequency noise is better correlated. A detailed study of the noise spectra dependence on the magnetic field for each individual NMOR system can provide a guideline for choosing optimal working parameters to reduce or eliminate the atomic excess noise.
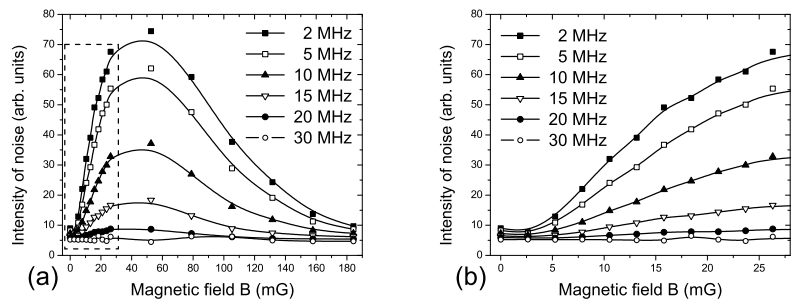

FIG. 5: (a) The level of noise at different frequencies is plotted as a function of the magnitude of the magnetic field. (b) The magnification of the dashed square region in (a). Different symbols denote different frequencies. Square: $2 \mathrm{MHz}$; hollow square: $5 \mathrm{MHz}$; triangle: $10 \mathrm{MHz}$; hollow triangle: $15 \mathrm{MHz}$; dot: $20 \mathrm{MHz}$; circle: $30 \mathrm{MHz}$. The solid lines are smooth connections of the data points.

To better demonstrate how the noise at a certain frequency depends on the magnitude of the magnetic field, we cut the three dimensional plots in Fig. 4 at specific frequencies along the plane of the magnetic field axis and the intensity axis. The cross sections picked up are shown in Fig. 5(a), in which the symbols square, hollow square, triangle, hollow triangle, dot and circle represent the level of noise at frequencies of $2 \mathrm{MHz}, 5 \mathrm{MHz}, 10$ $\mathrm{MHz}, 15 \mathrm{MHz}, 20 \mathrm{MHz}$ and $30 \mathrm{MHz}$, respectively. At the zero magnetic field, the noise is suppressed to the shot-noise level over the entire frequency region. For the frequencies lower than $20 \mathrm{MHz}$, the noise level increases quickly within about $50 \mathrm{mG}$, and comes back close to the shot-noise level within about $130 \mathrm{mG}$. For the frequencies higher than $20 \mathrm{MHz}$, the noise level essentially remains close to the shot-noise level. To show the details of the rising slope in the dashed square in Fig. 5(a), a magnification of this region is shown in Fig. 5(b). For low frequency noise, the noise level remains nearly minimum value not only at the zero magnetic field but also in a small region around zero magnetic field. In our data, the noise level of $2 \mathrm{MHz}$ and $5 \mathrm{MHz}$ noise is almost flat for magnetic fields ranging from 0 to $2.5 \mathrm{mG}$.

These results show that, for the best reduction of atomic excess noise in atom-optical applications, one should work near a zero longitudinal magnetic field. However, a rigorously exact zero magnetic field is not necessary, because the same reduction of noise can be obtained in a region around zero magnetic field. This 
property makes the implementation relatively easier and more reliable. As an example, to obtain the best reduction of the atomic excess noise in NMOR magnetometers, one might use an external calibrated magnetic field to compensate [5], so that longitudinal magnetic field is close to zero.

\section{CONCLUSION}

We have experimentally studied the noise spectra in a nonlinear magneto-optical rotation experiment in rubidium vapor. We have shown that a detailed study of noise reduction, due to the intensity correlation between two orthogonally polarized components of the laser beam, can suggest the optimal working conditions for reducing atomic excess noise. The noise in the difference signal of two orthogonal components at different frequencies has been studies as a function of magnetic field. The study of the noise dependence on both the noise frequency and the magnetic field shows that the maximum reduction of noise can be obtained around zero longitudinal magnetic field.

Our results can be used to reduce or eliminate atomic excess noise, and thus improve the sensitivity of NOMR magnetometers. The study also indicates the potential importance of the intensity correlations in other atomoptical applications such as atomic frequency references and the generation of squeezed light.

\section{ACKNOWLEDGEMENT}

We thank D. Budker, M.M. Kash, E.E. Mikhailov, F.A. Narducci, I. Novikova, D. Sarkisyan, G.R. Welch, and M.S. Zubairy for useful and fruitful discussions, and gratefully acknowledge the support from the Office of Naval Research, the Robert A. Welch Foundation (Grant \#A1261). One of us (T.S.V) also thanks NFSAT award TFP 2005/02 for financial support and the IQS and the Department of Physics of Texas A\&M University for their hospitality.
[1] E.B. Alexandrov, M.V. Balabas, A.S. Pasgalev, A.K. Vershovskii, and N.N. Yakobson, "Double-resonance atomic magnetometers: From gas discharge to laser pumping," Laser Phys. 6, 244 (1996).

[2] D. Budker, D.F. Kimball, S.M. Rochester, V.V. Yashchuk, and M. Zolotorev, "Sensitive magnetometry based on nonlinear magneto-optical rotation," Phys. Rev. A 62, 043403 (2000).

[3] V.A. Sautenkov, M.D. Lukin, C.J. Bednar, I, Novikova, E. Mikhailov, M. Fleischhauer, V.L. Velichansky, G.R. Welch, and M.O. Scully, "Enhancement of magneto-optic effects via large atomic coherence in optically dense media," Phys. Rev. A 62, 023810 (2000).

[4] I. K. Kominis, T. W. Kornack, J. C. Allred, and M. V. Romalis, "A subfemtotesla multichannel atomic magnetometer," Nature 422, 596 (2003).

[5] D. Budker, W. Gawlik, D.F. Kimball, S.M. Rochester, V. V. Yashchuk, and A. Weis, "Resonant nonlinear magneto-optical effects in atoms," Rev. Mod. Phys. 74, 1153 (2002).

[6] T. Yabuzaki, T. Mitsui, and U. Tanaka, "New type of high resolution sepctroscopy with a diode laser," Phys. Rev. Lett. 67, 2453 (1991).

[7] D.H. McIntyre, C.E. Fairchild, J. Cooper, and R. Walser, "Diode-laser noise spectroscopy of rubidium," Opt. Lett. 18, 1816 (1993).

[8] J.C. Camparo, "Conversion of laser phase noise to amplitude noise in an optically thick vapor," J. Opt. Soc. Am. B 15, 1177 (1998).

[9] J.C. Camparo and J.G. Coffer, "Conversion of laser phase noise to amplitude noise in a resonant atomic vapor: The role of laser linewidth," Phys. Rev. A 59, 728 (1999).

[10] R. J. Gehr, A. L. Gaeta, and R. W. Boyd, and G.S.Agarwal, "Excess noise acquired by a laser beam after propagating through an atomic-postassium vapor," Phys. Rev. A 51, 4152 (1995).
[11] R. Walser and P. Zoller, "Laser-noise-induced polarization fluctuation as a spectroscopic tool," Phys. Rev. A 49, 5067 (1994).

[12] J. Kitching, H.G. Robinson, L. Hollberg, S. Knappe, and R. Wynands, "Optical-pumping noise in laser-pumped, all-optical microwave frequency references," J. Opt. Soc. Am. B 18, 1676 (2001).

[13] G. Hétet, O. Glöckl, K.A. Pilypas, C.C. Harb, B.C. Buchler, H.-A. Bachor, and P.K. Lam, "Squeezed light for bandwidth-limited atom optics experiments at the rubidium D1 line," Journal of Physics B 40, 221 (2007).

[14] A.B. Matsko, I. Novikova, G.R. Welch, D. Budker, D.F. Kimball, and S.M. Rochester, "Vacuum squeezing in atomic media via self-rotation," Phys. Rev. A 66, 043815 (2002).

[15] J. Ries, B. Brezger, and A.I. Lvovsky, "Experimental vacuum squeezing in rubidium vapor via selfrotation," Phys. Rev. A 68, 025801 (2003).

[16] M.T.L. Hsu, G. Hétet, et. al. "Effect of atomic noise on optical squeezing via polariztion self-rotation in a thermal vapor cell," Phys. Rev. A 73, 023806 (2006).

[17] E.E. Mikhailov and I. Novikova, "Low-frequency vacuum squeezing via polarization self-rotation in Rb vapor," arXiv:0802.1558v1 (2008).

[18] V.A. Sautenkov, Y.V. Rostovtsev, and M.O. Scully, "Switching between photon-photon correlations and Raman anticorrelations in a coherently prepared Rb vapor," Phys. Rev. A 72, 065801 (2005).

[19] L.S. Cruz, D. Felinto, J.G. Aguirre Gómez, M. Martinelli, P. Valente, A. Lezama, and P. Nussenzveig, "Laser-noiseinduced correlations and anti-correlations in electromagnetically induced transparency," Eur. Phys. J. D 41, 531 (2007).

[20] V.A. Sautenkov, H. Li, Y.V. Rostovtsev, and M.O. Scully, "Power spectra and correlations of intensity fluctuations in electromagnetically induced transparency," J. 
Mod. Opt. 54, 2451 (2007).

[21] T.S. Varzhapetyan, H. Li, G.O. Ariunbold, V.A. Sautenkov, Y.V. Rostovtsev, and M.O. Scully, "Intensity correlations in resonance nonlinear magneto-opticcal rotation," arXiv:0803.3050v1 (2008).

[22] M. Martinelli, P. Valente, H. Failache, D. Felinto, L.S. Cruz, P. Nussenzveig, and A. Lezama, "Noise spec- troscopy of nonlinear magneto-optical resonances in $\mathrm{Rb}$ vapor," Phys. Rev. A 69, 043809 (2004).

[23] V.V. Vassiliev, S.A. Zibrov and V.L. Velichansky, "Compact extended-cavity diode laser for atomic spectroscopy and metrology" Rev. Sci. Instrum. 77, 013102 (2006). 